\title{
Asymptotic computation of Greeks under a stochastic volatility model
}

\author{
Sang-Hyeon Park ${ }^{1, a}$, Kiseop Lee ${ }^{b}$ \\ ${ }^{a}$ Daishin Securities, Korea; ${ }^{b}$ Department of Mathematics, University of Louisville, USA
}

\begin{abstract}
We study asymptotic expansion formulae for numerical computation of Greeks (i.e. sensitivity) in finance. Our approach is based on the integration-by-parts formula of the Malliavin calculus. We propose asymptotic expansion of Greeks for a stochastic volatility model using the Greeks formula of the Black-Scholes model. A singular perturbation method is applied to derive asymptotic Greeks formulae. We also provide numerical simulation of our method and compare it to the Monte Carlo finite difference approach.
\end{abstract}

Keywords: computation of Greeks, asymptotics, stochastic volatility, singular perturbation, Malliavin calculus

\section{Introduction}

Greeks are quantities representing partial derivatives of the price of a contingent claim. The Greeks are also called (risk) sensitivities since they describe movements of the price of a contingent claim with respect to risks. For example, the delta measures a rate of change of the option value with respect to the price change of the underlying asset (the first partial derivatives with respect to the underlying), and the vega is the derivative of the option value with respect to the volatility of the underlying asset. Greeks are very useful for derivatives traders, especially those who want to build a hedging portfolio. However, it is hard to obtain an explicit formula of a Greek in general financial models, including a stochastic volatility model.

Monte Carlo simulation for the finite difference approximation is a common approach to find Greeks. For instance, each sample of the Delta is estimated by $(1 / \epsilon)(P(t, x+\epsilon)-P(t, x))$ for a small $\epsilon$, where $P(t, x)$ is the price of the derivative at time $t$ and with the underlying value $x$. According to the Glasserman and Yao (1992) and Glynn (1989), the Monte Carlo simulation for the Greeks has a bad convergence rate and performs poorly if the payoff function is not smooth enough.

Another approach to calculate Greeks is the Malliavin calculus approach, which is introduced in Fournié et al. (1999, 2001). This approach, which we call the Malliavin Greeks, works well even when the payoff function is not continuous, such as a digital option.

However, the Malliavin Greeks approach requires the first variation process of the underlying to obtain Greeks. In general, the first variation process is given by the solution of the stochastic differential equation (SDE) associated with the underlying process. A few models such as BlackScholes (B.S.) have an explicit formula of their first variation process. The choice of the optimal weight is also an important issue in the Malliavin Greeks. Fournié et al. $(1999,2001)$ show that

\footnotetext{
${ }^{1}$ Corresponding author: Daishin Securities, 16, Gugjegeumyung-ro 8-gil, Yeongdeungpo-gu, Seoul 07330, Korea.

E-mail: laniean@daishin.com
}

Published 31 January 2016 / journal homepage: http://csam.or.kr

(c) 2016 The Korean Statistical Society, and Korean International Statistical Society. All rights reserved. 
an explicit optimal weight of the Black-Scholes model is easy to calculate; therefore, we can obtain efficient Malliavin Greeks under the Black-Scholes model.

A stochastic volatility model has been a popular alternative of the Black Scholes model. In a stochastic volatility model, the volatility is not a constant but another stochastic process and can describe various uncertain movements of volatility. Therefore, the stochastic volatility model became popular in finance. A typical stochastic volatility model assumes that the volatility is driven by a mean-reverting process. Hull and White (1987), Heston (1993), Fouque et al. (2000, 2003, 2011) are well known studies on stochastic volatility models. In particular, Fouque et al. (2000, 2003, 2011) derived an asymptotic solution for European options under a fast mean reverting stochastic volatility setting after observing a fast time scale volatility factor of mean reversion.

In this paper, we apply the Malliavin Greeks approach in Fournié et al. $(1999,2001)$ for an asymptotic expansion of the stochastic volatility model developed by Fouque et al. (2000, 2003, 2011). We derive Greeks formulae of a stochastic volatility model using the Black-Scholes model. The singular perturbation method and the Malliavin calculus are two main tools. We compare the finite difference approximation of Greeks to our results by numerical simulations.

Recently, Takahashi and Yamada $(2012,2014)$ studied the asymptotic expansions of stochastic volatility model. They present asymptotic expansions of option prices and Greeks that provided an error estimates around the Black-Scholes model in stochastic volatility model. The basic difference between our study and theirs is the underlying model. The stochastic volatility model developed by Fouque et al. $(2000,2003,2011)$ is a singular problem. Precisely, the volatility process of this model are diverged as the parameter of model goes to zero. However, Takahashi and Yamada $(2012,2014)$ studied regular cases, i.e. the volatility process are converged to a positive constant as the parameter of model goes to zero. Therefore, their approaches cannot be applied to the stochastic volatility model developed by Fouque et al. (2000, 2003, 2011).

The rest of the paper is organized as follows. In the Section 2, we briefly review the Malliavin calculus and results in Fournié et al. $(1999,2001)$. Section 3 presents asymptotic Greeks under the fast mean reversion model developed by Fouque et al. (2003). Numerical examples are provided in Section 4 that numerically verify the results in Section 3.

\section{Malliavin calculus and a computation of Greeks}

This section gives a brief review of the Malliavin calculus and the main results of Fournié et al. (1999, 2001). The Malliavin calculus introduces derivatives of the Wiener functional and can be presented as integration by part. We refer to Fournié et al. $(1999,2001)$ for details.

Let $\left\{W_{t}, 0 \leq t \leq T\right\}$ be an n-dimensional Brownian motion on a complete probability space $(\Omega, \mathcal{F}, P)$ with the natural filtration $\mathcal{F}_{t}$ generated by $W$. Let $F$ be a random variable of the form

$$
F=f\left(\int_{0}^{T} h_{1}(t) d W_{t}, \ldots, \int_{0}^{T} h_{n}(t) d W_{t}\right)
$$

where $f$ is an infinitely differentiable and rapidly decreasing function on $R^{n}$, and $h_{i} \in L^{2}([0, T] \times \Omega)$. The Malliavin derivative $D F$ is defined as the process $\left\{D_{t} F, t \geq 0\right\}$ of $L^{2}([0, T] \times \Omega)$

$$
D_{t} F=\sum_{i=1}^{n} \frac{\partial f}{\partial x_{i}}\left(\int_{0}^{T} h_{1}(t) d W_{t}, \ldots, \int_{0}^{T} h_{n}(t) d W_{t}\right) h_{i}(t), \quad t \geq 0 \text { a.s.. }
$$


We also define the norm for $F$ by

$$
\|F\|_{1,2}=\left(E\left[F^{2}\right]\right)^{\frac{1}{2}}+\left(E\left[\int_{0}^{T}\left(D_{t} F\right)^{2} d t\right]\right)^{\frac{1}{2}} .
$$

Let $\mathbb{D}^{1,2}$ denote the Banach space which is the completion of the set of $F$ with respect to the norm $\|\cdot\|_{1,2}$. Then $D$ is a closed linear operator from $\mathbb{D}^{1,2}$ to $L^{2}([0, T] \times \Omega)$. We now present some useful results in Fournié et al. (1999, 2001).

Proposition 1. Let $\phi: R^{n} \rightarrow R$ be a continuously differentiable function with bounded derivatives, and $F$ be a random vector in $\mathbb{D}^{1,2}$. Then $\phi(F) \in \mathbb{D}^{1,2}$ and

$$
D_{t} \phi(F)=\sum_{i=1}^{n} \frac{\partial \phi}{\partial x_{i}}(F) D_{t} F_{i}, \quad t \geq 0 \text { a.s.. }
$$

Proposition 1 presents a chain rule of Malliavin derivatives. The next proposition provides Malliavin derivatives of the solution of SDEs.

Proposition 2. Let $\left\{X_{t}, t \geq 0\right\}$ be an $R^{n}$ valued process driven by the $S D E$

$$
d X_{t}=\alpha\left(X_{t}\right) d t+\sigma\left(X_{t}\right) d W_{t},
$$

where $\alpha$ and $\sigma$ are continuously differentiable with bounded derivatives. Let $\left\{Y_{t}, t \geq 0\right\}$ be the associated first variation process defined by

$$
d Y_{t}=\alpha^{\prime}\left(X_{t}\right) Y_{t} d t+\sum_{i=1}^{n} \sigma_{i}^{\prime}\left(X_{t}\right) Y_{t} d W_{t}^{i}, \quad Y_{0}=I_{n}
$$

where $I_{n}$ is the identity matrix of $R^{n}$, primes denote derivatives, and $\sigma_{i}$ is the $i$-th column vector of $\sigma$. We also assume the diffusion matrix $\sigma$ satisfies

$$
\xi^{T} \sigma^{T}(x) \sigma(x) \xi \geq c|\xi|^{2}, \quad \text { for any } \xi, x \in R^{n},
$$

for some $c>0$. Then the process $\left\{X_{t}, t \geq 0\right\}$ belongs to $\mathbb{D}^{1,2}$ and its Malliavin derivative is given by

$$
D_{s} X_{t}=Y_{t}\left(Y_{s}\right)^{-1} \sigma\left(X_{s}\right) 1_{s \leq t}, \quad s \geq 0 \text { a.s.. }
$$

Therefore, If $\phi \in C_{b}^{1}\left(R^{n}\right)$ then we have

$$
D_{s} \phi\left(X_{t}\right)=\nabla \phi\left(X_{t}\right) Y_{t}\left(Y_{s}\right)^{-1} \sigma\left(X_{s}\right) 1_{s \leq t}, \quad s \geq 0 \text { a.s. }
$$

and

$$
D_{s} \int_{0}^{T} \phi\left(X_{t}\right) d t=\int_{s}^{T} \nabla \phi\left(X_{t}\right) Y_{t}\left(Y_{s}\right)^{-1} \sigma\left(X_{s}\right) d t, \quad \text { a.s. }
$$

We now review the Skorohod integral, which is associated with Malliavin derivatives.

Definition 1. We say that $u \in \operatorname{Dom}(\delta)$, if

$$
E\left[\int_{0}^{T} u_{t} D_{t} \phi d t\right]<\infty
$$


for a stochastic process $u$ and for any $\phi \in \mathbb{D}^{1,2}$. Let us define the Skorohod integral $\delta(u):=\int_{0}^{T} u_{t} \delta W_{t}$ by

$$
E\left[\int_{0}^{t} u_{t} D_{t} \phi d t\right]=E[\phi \delta(u)], \text { for all } \phi \in \mathbb{D}^{1,2} .
$$

Actually, $\operatorname{Dom}(\delta)$ contains $\mathcal{F}_{t}$-adapted processes which belongs to $L^{2}([0, T] \times \Omega)$; In this case, the divergence operator $\delta$ coincides with Ito integral,

$$
\int_{0}^{T} u_{t} \delta W_{t}=\int_{0}^{T} u_{t} d W_{t}
$$

Next proposition presents an integration by part formula for Malliavin derivatives.

Proposition 3. Let $F$ be a $\mathcal{F}_{t}$-adapted random variable in $\mathbb{D}^{1,2}$. Then for any $u \in \operatorname{Dom}(\delta)$ we have

$$
\delta(F u)=F \delta(u)-\int_{0}^{T} D_{t} F u_{t} d t .
$$

Proposition 4 is a called the Clark-Ocone formula and that is very useful. It expresses the value of the function $F$ as the sum of its mean value and an Itô integral with respect to Malliavin derivatives.

Proposition 4. Let $F$ be a random variable in $\mathrm{D}^{1,2}$. Then we have

$$
F=E[F]+\int_{0}^{T} E\left[D_{t} F \mid \mathcal{F}_{t}\right] d W_{t} .
$$

Proposition 5. For any fixed $t \in[0, T]$ and $u \in \operatorname{Dom}(\delta)$, we obtain $1_{(0, t]} u \in \operatorname{Dom}(\delta)$ and

$$
\begin{aligned}
\int_{0}^{t} u_{s} \delta W_{s} & =\int_{0}^{T} 1_{(0, t]} u_{s} \delta W_{s}, \\
\int_{0}^{t} u_{s} \delta W_{s}+\int_{t}^{T} u_{s} \delta W_{s} & =\int_{0}^{T} u_{s} \delta W_{s} .
\end{aligned}
$$

\subsection{Greeks and Malliavin calculus}

We assume that the underlying asset is the solution of the stochastic differential equation

$$
d X_{t}=\alpha\left(X_{t}\right) d t+\sigma\left(X_{t}\right) d W_{t},
$$

which is the special case when $n=1$ in Proposition 2. We now consider a European option pricing problem under the risk neutral measure $\mathbb{Q}$

$$
P_{X}(t, x)=E^{\mathbb{Q}}\left[e^{-r(T-t)} h\left(X_{T}\right) \mid X_{t}=x\right],
$$

where the payoff function $h$ belongs to $L^{2}(R)$. We use the notation $E^{X_{t}}[\cdot]:=E^{\mathbb{Q}}\left[\cdot \mid X_{t}=x\right]$ for convenience.

Let us study a Malliavin Greeks formula of a European option. The next theorem presents the delta of a European option price. 
Theorem 1. Let a $(\tau)$ be a continuous deterministic function. Suppose that

$$
\int_{t}^{T} a(\tau) d \tau=1
$$

for a fixed $T>t$. Then we have

$$
\begin{aligned}
\frac{\partial}{\partial x}\left(E^{X_{t}}\left[h\left(X_{T}\right)\right]\right) & =E^{X_{t}}\left[h\left(X_{T}\right) \pi^{\Delta}\right], \\
\pi^{\Delta} & :=\int_{t}^{T} a(s) \frac{Y_{s}}{\sigma\left(X_{s}\right)} d W_{s},
\end{aligned}
$$

where $Y_{t}$ is the first variation process of $X_{t}$.

Proof: Refer to the proof of the Proposition 3.2 in Fournié et al. (1999).

Remark 1. According to Fournié et al. (1999), the payoff function $h$ does not need to be smooth to guarantee the efficient convergence rate of Malliavin Greeks. Using the same argument in Theorem 1, we can derive other Greeks such as Gamma(the second derivatives w.r.t. the price) and Vega(the derivatives w.r.t. $\sigma$ ). We refer to Fournié et al. (1999) for details.

\section{Fast mean-reverting OU volatility model and singular perturbation method}

In this section, we a study stochastic volatility model with volatility driven by a fast mean-reverting Ornstein-Uhlenbeck process. The fast mean reverting stochastic volatility model is first developed by Fouque et al. (2003) and is an application of the asymptotic analysis in option pricing. This stochastic volatility model leads to an asymptotic option price by the ergodic theorem. Consequently, they derived an option price of a fast mean-reverting stochastic volatility model from the option price of the Black-Sholes model.

We study the same fast mean-reverting stochastic volatility model, and study how to calculate Greeks borrowing a similar idea of adopting the Black-Scholes model. Under a risk-neutral measure, the underlying price $X_{t}$ of the fast mean reverting stochastic volatility model satisfies SDE

$$
\begin{aligned}
& \frac{d X_{t}}{X_{t}}=r d t+f\left(V_{t}\right) d W_{t}^{(0)}, \\
& d V_{t}=\left[\frac{1}{\epsilon}\left(m-V_{t}\right)-\frac{\sqrt{2} v}{\sqrt{\varepsilon}} \Lambda\left(V_{t}\right)\right] d t+\frac{\sqrt{2} v}{\sqrt{\varepsilon}} d W_{t}^{(1)},
\end{aligned}
$$

where $W_{t}^{(0)}$ and $W_{t}^{(1)}$ are dependent one dimensional Brownian motions with correlated coefficient $\rho$, and $\Lambda(v)$ is given by

$$
\Lambda(v)=\rho \frac{(\mu-r)}{f(v)}+\gamma(v) \sqrt{1-\rho^{2}} .
$$

Here, the market price of risk $\gamma(v)$ is determined by Girsanov theorem. Constants $\mu$ and $v^{2}$ are the mean and the variance of the invariant distribution $V_{\infty}$, respectively. We assume that $\epsilon$ is small enough, which means that the volatility process $V(t)$ is fast mean-reverting.

Under this formulation, the option price

$$
C(t, x, v)=E^{\mathbb{Q}}\left[e^{-r(T-t)} h\left(X_{T}\right) \mid X_{t}=x, V_{t}=v\right]
$$


satisfies the partial differential equation (PDE),

$$
\left[\frac{1}{\epsilon} \mathcal{L}_{0}+\frac{1}{\sqrt{\epsilon}} \mathcal{L}_{1}+\mathcal{L}_{2}\right] C=0
$$

where

$$
\begin{aligned}
& \mathcal{L}_{0}=(m-v) \frac{\partial}{\partial v}+v^{2} \frac{\partial^{2}}{\partial v^{2}} \\
& \mathcal{L}_{1}=-\sqrt{2} v \Lambda(v) \frac{\partial}{\partial v}+\sqrt{2} v \rho f(v) x \frac{\partial^{2}}{\partial x \partial v} \\
& \mathcal{L}_{2}=\frac{\partial}{\partial t}-r \bullet+r x \frac{\partial}{\partial x}+\frac{1}{2} f^{2}(v) x^{2} \frac{\partial^{2}}{\partial x^{2}}
\end{aligned}
$$

with the terminal condition of $C(T, x, y)=h(x)$. Let $C=\sum_{n=0}^{\infty} \epsilon^{n / 2} C_{n}$, then the PDE (3.3) becomes

$$
\left[\frac{1}{\epsilon} \mathcal{L}_{0}+\frac{1}{\sqrt{\epsilon}} \mathcal{L}_{1}+\mathcal{L}_{2}\right] C=\frac{1}{\epsilon}\left[\mathcal{L}_{0} C_{0}\right]+\frac{1}{\sqrt{\epsilon}}\left[\mathcal{L}_{1} C_{0}+\mathcal{L}_{0} C_{1}\right]+\left[\mathcal{L}_{2} C_{0}+\mathcal{L}_{0} C_{2}+\mathcal{L}_{1} C_{1}\right]+\cdots=0 .
$$

By the solvability condition (refer to Fouque et al. (2003)), the leading order term $C_{0}$ and the first correction term $C_{1}$ are independent of $v$. Moreover, $C_{0}$ and $C_{1}$ satisfy partial differential equations

$$
\begin{aligned}
\left\langle\mathcal{L}_{2}\right\rangle C_{0}(t, x) & =0, \\
C_{0}(T, x) & =h(x)
\end{aligned}
$$

and

$$
\begin{aligned}
\left\langle\mathcal{L}_{2}\right\rangle C_{1}(t, x) & =\left\langle\mathcal{L}_{1} \mathcal{L}_{0}^{-1}\left(\mathcal{L}_{2}-\left\langle\mathcal{L}_{2}\right\rangle\right)\right\rangle C_{0}=-\left(A_{1}-2 A_{2}\right) x^{2} \frac{\partial^{2} C_{0}}{\partial x^{2}}+A_{2} x^{3} \frac{\partial^{3} C_{0}}{\partial x^{3}} \\
C_{1}(T, x) & =0
\end{aligned}
$$

respectively. Here, $A_{1}=(v / \sqrt{2})\left\langle\phi^{\prime} \Lambda\right\rangle$ and $A_{2}=(\rho v / \sqrt{2})\left\langle\phi^{\prime} f\right\rangle$. $\phi$ is the solution of $\mathcal{L}_{0} \phi(v)=$ $f^{2}(v)-\left\langle f^{2}\right\rangle$. The bracket $\langle\cdot\rangle$ denotes the expectation with respect to the invariant distribution of $Y$ defined by

$$
\langle g\rangle=\int g(v) \Phi(v) d v, \quad \text { and } \quad \Phi(v):=\frac{1}{\sqrt{2 \pi v^{2}}} e^{-\frac{(v-m)^{2}}{2 v^{2}}} .
$$

Note that the operator $\left\langle\mathcal{L}_{2}\right\rangle$ is exactly same to the operator $\mathcal{L}_{2}$ by replacing $f(v)$ with the constant $\bar{\sigma}=\sqrt{\left\langle f^{2}\right\rangle}$.

We now discuss the Delta formula of $C(t, x, v)$ driven by Malliavin derivatives.

Theorem 2. Let $S_{t}$ be the solution of the following SDE:

$$
d S_{t}=r S_{t} d t+\bar{\sigma} S_{t} d W_{t}^{(0)},
$$

with the initial condition $S_{t}=s:=x$. Then, we have an approximation formula $C=C_{0}+\epsilon^{1 / 2} C_{1}+O(\epsilon)$, where

$$
\begin{aligned}
C_{0}(t, x) & =E^{S_{t}}\left[e^{-r(T-t)} h\left(S_{T}\right)\right], \\
C_{1}(t, x) & =E^{S_{t}}\left[\int_{t}^{T} e^{-r(s-t)} g\left(s, S_{s}\right) d s\right], \\
g(t, x) & :=\left(A_{1}-2 A_{2}\right) x^{2} \frac{\partial^{2} C_{0}}{\partial x^{2}}-A_{2} x^{3} \frac{\partial^{3} C_{0}}{\partial x^{3}} .
\end{aligned}
$$


Moreover, (3.5) leads to

$$
\frac{\partial C(t, x)}{\partial x}=E^{S_{t}}\left[e^{-r(T-t)} h\left(S_{T}\right) \pi_{0}^{\Delta}(T-t, x)\right]+\epsilon^{\frac{1}{2}} E^{S_{t}}\left[\int_{t}^{T} e^{-r(s-t)} g\left(s, S_{s}\right) \pi_{1}^{\Delta}(s-t, x) d s\right]+O(\epsilon)
$$

where $\pi_{0}^{\Delta}(T-t, x)=W_{T-t} /\{x \bar{\sigma}(T-t)\}$ and $\pi_{1}^{\Delta}(s-t, x)=W_{s-t} /\{x \bar{\sigma}(s-t)\}$.

Proof: The infinitesimal generator of $S_{t}$ is

$$
r x \frac{\partial}{\partial x}+\frac{1}{2} \bar{\sigma}^{2} x^{2} \frac{\partial^{2}}{\partial x^{2}}
$$

Therefore, we have a probabilistic representation of the solution by the Feyman-Kac theorem. Since $S_{t}$ follows the Black-Scholes model, we obtain an explicit formula for the leading term, $\partial C_{0} / \partial x$, by applying Theorem 1 and elementary calculation of the first variation process of $S_{t}$. (We choose a deterministic function $a(\tau)$ in Theorem 1 as $1 /(T-t)$. According to Fouque et al. (2003), $C=$ $C_{0}+\epsilon^{1 / 2} C_{1}+O(\epsilon)$ and terms $C_{0}$ and $C_{1}$ are smooth and bounded. Thus,

$$
\frac{\partial}{\partial x} E^{S_{t}}\left[\int_{t}^{T} g\left(s, S_{s}\right) d s\right]=\int_{t}^{T} \frac{\partial}{\partial x} E^{S_{t}}\left[g\left(s, S_{s}\right)\right] d s .
$$

To apply Theorem 1 , we take $a(\tau)=1 /(s-t)$ i.e. $\int_{t}^{s} a(\tau) d \tau=1$. Then we get (3.6).

Remark 2. By the integration-by-part formula (refer to Fournié (1999)), we have representations of Gamma and Vega in the following.

$$
\begin{gathered}
\frac{\partial^{2} C(t, x)}{\partial x^{2}}=E^{S_{t}}\left[e^{-r(T-t)}\left(S_{T}-K\right)^{+} \pi_{0}^{\gamma}(T-t, x)\right]+\epsilon^{\frac{1}{2}} E^{S_{t}}\left[\int_{t}^{T} e^{-r(s-t)} g\left(s, S_{s}\right) \pi_{1}^{\gamma}(s-t, x) d s\right]+O(\epsilon), \\
\frac{\partial C(t, x)}{\partial \bar{\sigma}}=E^{S_{t}}\left[e^{-r(T-t)}\left(S_{T}-K\right)^{+} \pi_{0}^{\bar{\sigma}}(T-t, x)\right]+\epsilon^{\frac{1}{2}} E^{S_{t}}\left[\int_{t}^{T} e^{-r(s-t)} g\left(s, S_{s}\right) \pi_{0}^{\sigma}(s-t, x) d s\right]+O(\epsilon),
\end{gathered}
$$

where

$$
\begin{aligned}
\pi_{0}^{\gamma}(T-t, x) & :=\frac{1}{x^{2} \bar{\sigma}(T-t)}\left(\frac{\left(W_{T-t}^{(0)}\right)^{2}}{\bar{\sigma}(T-t)}-W_{T-t}^{(0)}-\frac{1}{\bar{\sigma}}\right), \\
\pi_{1}^{\gamma}(s-t, x) & :=\frac{1}{x^{2} \bar{\sigma}(s-t)}\left(\frac{\left(W_{s-t}^{(0)}\right)^{2}}{\bar{\sigma}(s-t)}-W_{s-t}^{(0)}-\frac{1}{\bar{\sigma}}\right), \\
\pi_{0}^{\bar{\sigma}}(T-t, x) & :=\frac{\left(W_{T-t}^{(0)}\right)^{2}}{\bar{\sigma}(T-t)}-W_{T-t}^{(0)}-\frac{1}{\bar{\sigma}} \\
\pi_{1}^{\bar{\sigma}}(s-t, x) & :=\frac{\left(W_{s-t}^{(0)}\right)^{2}}{\bar{\sigma}(s-t)}-W_{s-t}^{(0)}-\frac{1}{\bar{\sigma}} .
\end{aligned}
$$

Under this stochastic volatility model, a direct application of Fournié et al. $(1999,2001)$ leads a bad convergence rate in the numerical simulation of Malliavin Greeks. Since the model is fast-mean reverting i.e., $1 / \epsilon$ is sufficiently large, the first variation process and an optimal weight of (3.1) should 
have large variances. This implies that random variables in Malliavin formulas, such as $h\left(X_{T}\right) \pi^{\Delta}$ of (2.17), also have large variances. Therefore, the Malliavin Greeks have a low level convergence rate in numerical simulation. Moreover, the volatility function $f(v)$ which is estimated by market data may not guarantee the existence of the first variation process.

However, the above asymptotic approach does not contain the volatility function $f(v)$ and the large coefficient $1 / \epsilon$. We only need constants $\bar{\sigma}=\sqrt{\left\langle f^{2}\right\rangle}$ and $A_{1,2}$, not $f(v)$ nor $1 / \epsilon$. This implies that the variance of a random process $e^{-r(T-t)} h\left(S_{T}\right) \pi_{0}^{\Delta}(T-t, x)$ in (3.6) is adequate for numerical simulation.

Next, we rewrite the formula (3.6) by replacing partial derivative terms $\partial^{2} C_{0} / \partial x^{2}$ and $\partial^{3} C_{0} / \partial x^{3}$ with alternative representations. In a European Call and a Put option cases, we already know explicit formulae for the Gamma and the Speed ( $3^{\text {rd }}$ derivatives with respect to underlying). However, they are generally unknown in other cases. Therefore, we need to find a suitable representation with respect to high order partial derivatives such as Gamma and Speed which appear in the correction terms. By applying the Black-Scholes model, we have the following.

Theorem 3. The first correction term $E^{S_{t}}\left[\int_{t}^{T} e^{-r(s-t)} g\left(s, S_{s}\right) \pi_{1}^{\Delta}(s-t, x) d s\right]$ of the formula (3.6) is reformulated by

$$
E^{S_{t}}\left[H\left(S_{T}\right) \int_{t}^{T} e^{-r(s-t)} \Gamma\left(T, t, x, s, S_{s}\right) d s\right],
$$

where $H(x)=e^{-r(T-t)}(x-K)^{+}$and

$$
\begin{aligned}
\Gamma\left(T, t, x, s, S_{s}\right):= & \pi_{1}^{\Delta}(s-t, x)\left(\left(A_{1}+2 A_{2}\right) S_{s}^{2} \pi_{0}^{\gamma}\left(T-s, S_{s}\right)+A_{2} S_{s}^{3} \pi_{0}^{\mathcal{S}}\left(T-s, S_{s}\right)\right) \\
\pi_{0}^{\mathcal{S}}(T-t, x):= & \frac{\left(W_{T-t}^{(0)}\right)^{3}}{x^{2} \bar{\sigma}^{2}(T-t)^{2}}-\left(W_{T-t}^{(0)}\right)^{2}\left(\frac{2}{x^{3} \bar{\sigma}^{2}(T-t)^{2}}+\frac{1}{x^{2} \bar{\sigma}^{2}(T-t)}\right) \\
& +W_{T-t}^{(0)}\left(\frac{2}{x^{3} \bar{\sigma}(T-t)}-\frac{2}{x^{2} \bar{\sigma}^{2}(T-t)}-\frac{1}{\bar{\sigma}}\right)+\frac{2}{x^{3} \bar{\sigma}^{2}(T-t)}+\frac{1}{x^{2} \bar{\sigma}} .
\end{aligned}
$$

Proof: First, we show that $\partial^{3} C_{0} / \partial x^{3}=E^{S_{t}}\left[H\left(S_{T}\right) \pi_{0}^{\mathcal{S}}(T-t, x)\right]$. Let $S_{T}^{x}=x+\int_{t}^{T} r S_{s} d s+\int_{t}^{T} \bar{\sigma} S_{s} d W_{s}^{(0)}$, which means that the process $S_{T}$ starts from $x$ at time $t$. By the integration by part formula for gamma, we have

$$
\begin{aligned}
\frac{\partial^{3} C_{0}}{\partial x^{3}} & =\frac{\partial}{\partial x} \frac{\partial^{2} C_{0}}{\partial x^{2}}=\frac{\partial}{\partial x} E^{S_{t}}\left[H\left(S_{T}^{x}\right) \pi_{0}^{\gamma}(T-t, x)\right] \\
& =E^{Q}\left[\frac{\partial H\left(S_{T}^{x}\right)}{\partial x} \pi_{0}^{\gamma}(T-t, x)+H\left(S_{T}^{x}\right) \frac{\partial \pi_{0}^{\gamma}}{\partial x}(T-t, x) \mid S_{t}=x\right] \\
& =E^{Q}\left[H^{\prime}\left(S_{T}^{x}\right) \frac{\partial S_{T}^{x}}{\partial x} \pi_{0}^{\gamma}(T-t, x) \mid S_{t}=x\right]+E^{Q}\left[H\left(S_{T}^{x}\right) \frac{\partial \pi_{0}^{\gamma}}{\partial x}(T-t, x) \mid S_{t}=x\right] .
\end{aligned}
$$

Using the integration by part formula, we have

$$
\begin{aligned}
E^{Q}\left[H^{\prime}\left(S_{T}^{x}\right) \frac{\partial S_{T}^{x}}{\partial x} \pi_{0}^{\gamma}(T-t, x) \mid S_{t}=x\right] & =E^{Q}\left[\int_{t}^{T} D_{s} H\left(S_{T}^{x}\right) \frac{\pi_{0}^{\gamma}(T-t, x)}{x \bar{\sigma}(T-t)} d s \mid S_{t}=x\right] \\
& =E^{Q}\left[H\left(S_{T}^{x}\right) \int_{t}^{T} \frac{\pi_{0}^{\gamma}(T-t, x)}{x \bar{\sigma}(T-t)} \delta W_{s}^{(0)} \mid S_{t}=x\right] .
\end{aligned}
$$




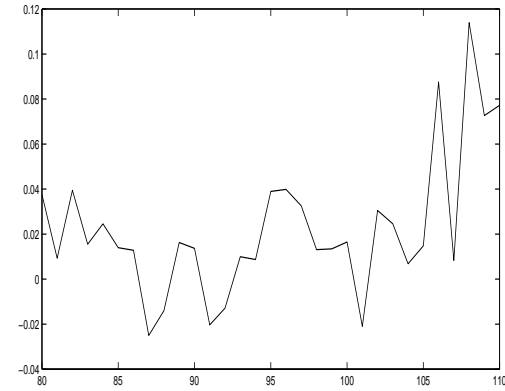

Figure 1: Error of call option price.

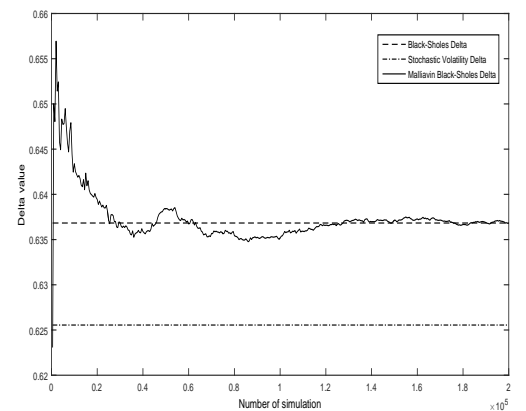

Figure 2: Malliavin Delta of leading term $\partial C_{0} / \partial X_{t}$ : Delta of Black-Sholes model at $X_{t}=K$.

Using the Wiener-Itô Chaos expansion to calculate the term $\int_{t}^{T}\left\{\pi_{0}^{\gamma}(T-t, x)\right\} /\{x \bar{\sigma}(T-t)\} \delta W_{s}^{(0)}$, we can check that

$$
E^{Q}\left[H\left(S_{T}^{x}\right)\left(\int_{t}^{T} \frac{\pi_{0}^{\gamma}(T-t, x)}{x \bar{\sigma}(T-t)} \delta W_{s}^{(0)}+\frac{\partial \pi_{0}^{\gamma}}{\partial x}(T-t, x)\right) \mid S_{t}=x\right]=E^{S_{t}}\left[H\left(S_{T}\right) \pi_{0}^{\mathcal{S}}(T-t, x)\right] .
$$

However, the strong Markov property of $S_{t}, t \leq s \leq T$ leads to

$$
\begin{aligned}
E^{S_{t}} & {\left[g\left(s, S_{s}\right) \pi_{1}^{\Delta}(s-t, x)\right] } \\
= & E^{Q}\left[\left\{\left(A_{1}-2 A_{2}\right) S_{s}^{2} \frac{\partial^{2} C_{0}}{\partial x^{2}}\left(s, S_{s}\right)-A_{2} S_{s}^{3} \frac{\partial^{3} C_{0}}{\partial x^{3}}\left(s, S_{s}\right)\right\} \pi_{1}^{\Delta}(s-t, x) \mid S_{t}=x\right] \\
= & E^{Q}\left[\left(A_{1}-2 A_{2}\right) S_{s}^{2} E^{Q}\left[H\left(S_{T}\right) \pi_{0}^{\gamma}\left(T-s, S_{s}\right) \mid S_{s}\right] \pi_{1}^{\Delta}(s-t, x) \mid S_{t}=x\right] \\
& -E^{Q}\left[A_{2} S_{s}^{3} E^{Q}\left[H\left(S_{T}\right) \pi_{0}^{\mathcal{S}}\left(T-s, S_{s}\right) \mid S_{s}\right] \pi_{1}^{\Delta}(s-t, x) \mid S_{t}=x\right] \\
= & E^{Q}\left[E^{Q}\left[\left(A_{1}-2 A_{2}\right) S_{s}^{2} H\left(S_{T}\right) \pi_{0}^{\gamma}\left(T-s, S_{s}\right) \pi_{1}^{\Delta}(s-t, x) \mid S_{s}\right] \mid S_{t}=x\right] \\
& -E^{Q}\left[E^{Q}\left[A_{2} S_{s}^{3} H\left(S_{T}\right) \pi_{0}^{\mathcal{S}}\left(T-s, S_{s}\right) \pi_{1}^{\Delta}(s-t, x) \mid S_{s}\right] \mid S_{t}=x\right] \\
= & E^{Q}\left[\left(A_{1}-2 A_{2}\right) S_{s}^{2} H\left(S_{T}\right) \pi_{0}^{\gamma}\left(T-s, S_{s}\right) \pi_{1}^{\Delta}(s-t, x) \mid S_{t}=x\right] \\
& -E^{Q}\left[A_{2} S_{s}^{3} H\left(S_{T}\right) \pi_{0}^{\mathcal{S}}\left(T-s, S_{s}\right) \pi_{1}^{\Delta}(s-t, x) \mid S_{t}=x\right] .
\end{aligned}
$$

By Fubini's theorem, we get the result.

\section{Numerical results}

In this section, we present numerical performance of our method. We consider a European call option with the exercise value $K=100$ and the expiry date $T-t=1$. Under a risk-neutral measure, we assume that the underlying $X_{t}$ satisfies (3.1). Let $f(v)=0.2 * e^{v}$ and $\Lambda(v)=0$. Set $r=0.04$, $\epsilon=0.01, m=0, v=0.4$, and $\rho=0$. In this setting, we compare the Malliavin Delta of (3.6) at $X_{t}=K=100$ to a finite difference Delta $\{u(x+\Delta x)-u(x-\Delta x)\} /(2 \Delta x)$. We also compute an asymptotic delta which is given by Fouque et al. $(2000,2003,2011)$ as the reference value since we do not know an exact formula of the Delta under the model (3.1). First, we simulate the price difference between an asymptotic option price and the Monte-Carlo simulation price. As in Figure 1, the asymptotic option price has $O(\sqrt{\epsilon})$ error, within 0.05 . This implies that our test performs well for 


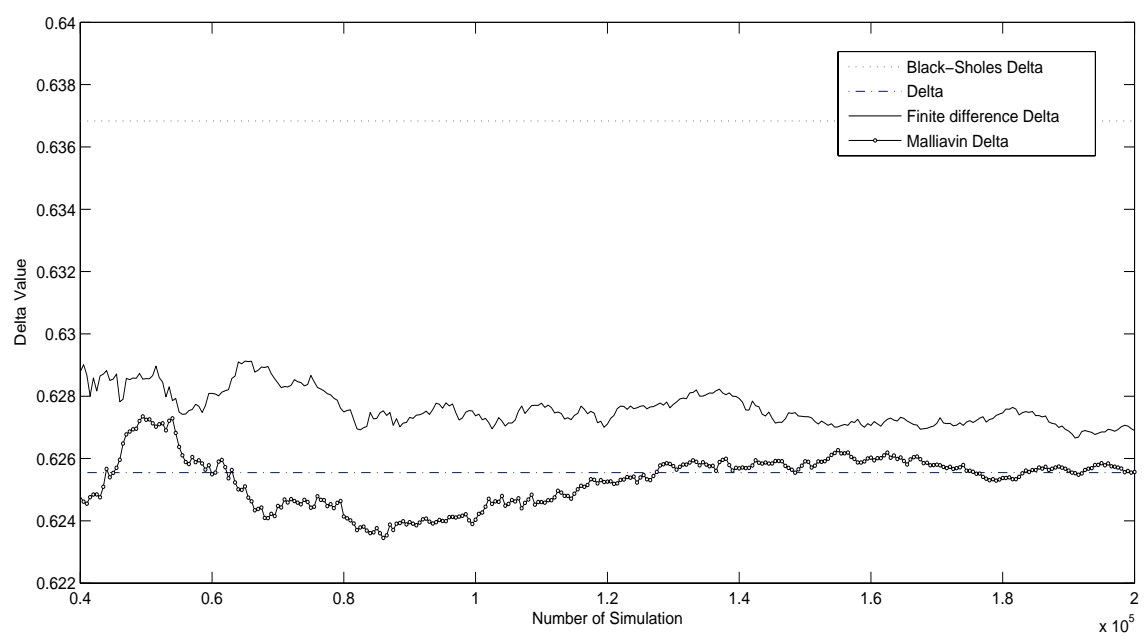

Figure 3: Call option Delta, $X_{t}=K$.

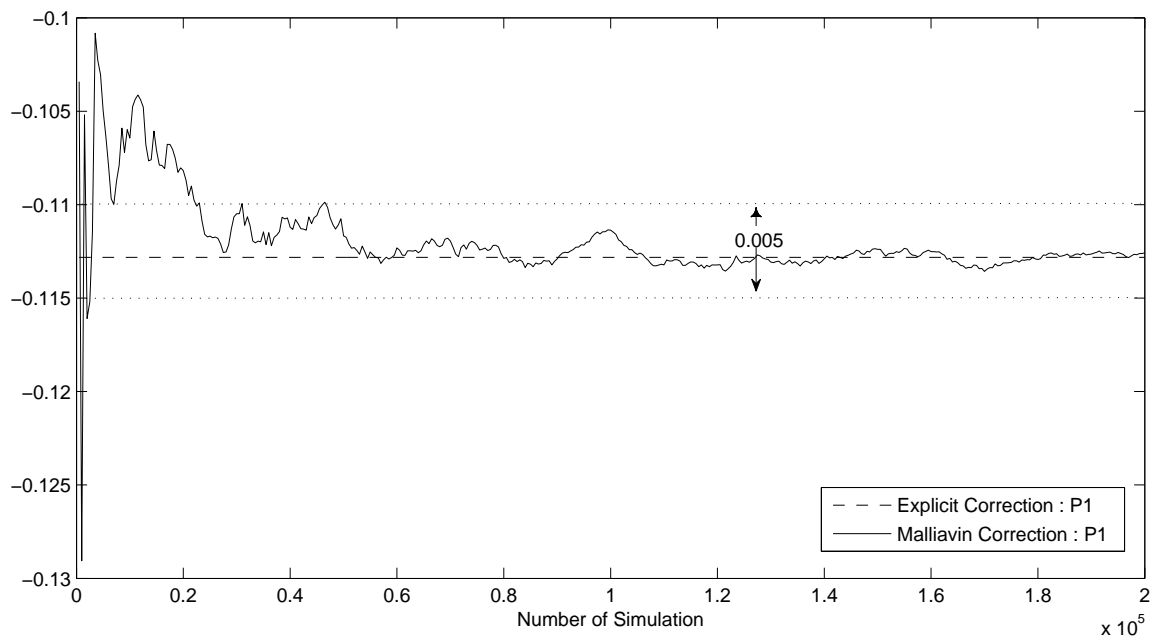

Figure 4: First correction: $\partial C_{1} / \partial X_{t}, X_{t}=K$.

comparing Delta of (3.6) to that of finite difference method. Figure 2 presents a Malliavin Delta of the Black-Scholes model. According to Figure 2, Malliavin Delta requires $1.2 \cdot 10^{5}$ times simulations for sufficient approximation. Figure 3 shows that the reference Delta under the stochastic volatility is close to 0.626 . Unfortunately, a finite difference delta has a bad convergence performance. However, Malliavin approach needs $1.2 \cdot 10^{5}$ simulations to obtain $0.04 \%$ accuracy. Therefore, we can reduce the time cost by applying an our method.

Figures 2 and 4 imply that the accuracy of the Malliavin Delta under the stochastic volatility model depends on the accuracy of leading term $\partial C_{0} / \partial X_{t}$. Figure 4 shows the accuracy of the first correction $\partial C_{1} / \partial X_{t}$. We notice that $\sqrt{\epsilon} \partial C_{0} / \partial X_{t}$ also requires $2 \cdot 10^{4}$ times simulations to reach a 


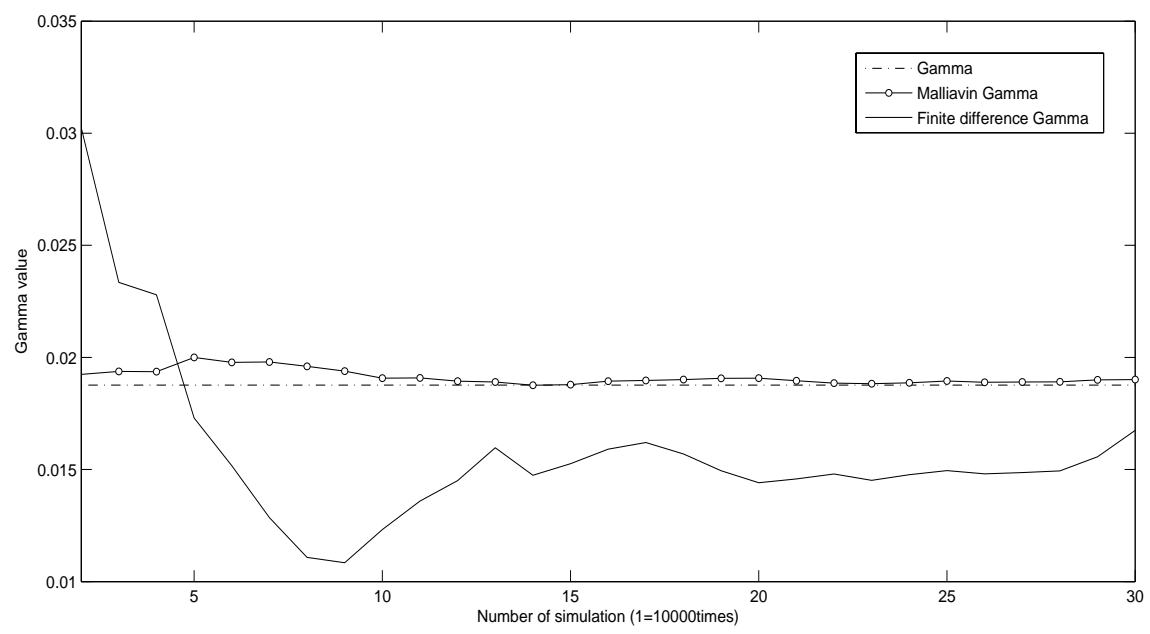

Figure 5: Call option Gamma, $X_{t}=K$.

suitable accuracy. Therefore, we can obtain proper approximation, if Malliavin Greeks of the leading term $\partial C_{0} / \partial X_{t}$ has less error. In this point of view, the study of leading term Greeks is also an issue in this field. It will be discussed in future work.

Finally, we compare the second derivative Gamma driven by Malliavin calculus to a finite difference Gamma. In Figure 5, we present a finite difference Gamma and Malliavin Gamma under the same setting. Here, we use the formula $\{C(x+\Delta x)+C(x-\Delta x)-2 C(x)\} /(\Delta x)^{2}$ for the finite difference Gamma. Likewise the finite difference Gamma in Figure 5, finite difference second derivatives are unstable in general. However, Malliavin Gamma has a similar convergence rate with respect to the Delta. Therefore, the Malliavin approach is efficient to compute high order derivatives.

\section{Conclusion}

In this paper, we studied an asymptotic computation method of Greeks under a stochastic volatility model. We utilized the Malliavin calculus and the singular perturbation method to derive a asymptotic formula of Greeks. Based on the Black-Scholes model, Greeks of stochastic volatility model are decomposed by two terms, the Greek of constant volatility model and a correction term. We also presented numerical simulations for the validation of the result. This simulation result shows that asymptotic Greeks has efficient performance rather than finite difference approximation. Especially, asymptotic Greeks provides better performance in the case of the Gamma simulation. This approach provides a stable computation method than the finite difference approximation for Greeks under the stochastic volatility model.

\section{References}

Fournié E, Lasry JM, Lebuchoux J, and Lions PL (2001). Applications of Malliavin calculus to Monte Carlo methods in finance II, Finance Stochastics, 5, 201-236

Fournié E, Lasry JM, Lebuchoux J, Lions PL, and Touzi N (1999). Applications of Malliavin calculus to Monte Carlo methods in finance, Finance Stochastics, 3, 391-412. 
Fouque JP, Papanicolaou G, and Sircar R (2000). Derivatives in Financial Markets with Stochastic Volatility, Cambridge University Press.

Fouque JP, Papanicolaou G, and Sircar R (2003). Singular perturbations in option pricing, SIAM Journal on Applied Mathematics, 63, 1648-1665.

Fouque JP, Papanicolaou G, Sircar R and Solna K (2011). Multiscale Stochastic Volatility for Equity, Interest-Rate and Credit Derivatives, Cambridge University Press.

Glasserman P and Yao DD (1992). Some guideline and guarantees for common random number, Management Science, 38, 884-908.

Glynn PW (1989). Optimization of stochastic system via simulation, In Proceedings of the 1989 Winter simulation Conference. San Diego: Society for Computer Simulation, 90-105.

Heston S (1993). A closed-form solution for options with stochastic volatility with applications to bond and currency options, Review of Financial Studies, 6, 327-343.

Hull J and White A (1987). The pricing of options on assets with stochastic volatility, The Journal of Finance, 42, 281-300.

Takahashi A and Yamada T (2012). An asymptotic expansion with push-down of Malliavin weights, SIAM Journal on Financial Mathematics, 3, 95-136.

Takahashi A and Yamada T (2014). On error estimates for asymptotic expansions with Malliavin weights: application to stochastic volatility model, Mathematics of Operations Research, 513541.

Received August 16, 2015; Revised December 3, 2015; Accepted December 5, 2015 